\title{
Metal ion release from TiN coated CoCrMo orthopedic implant material
}

\author{
Uğur Türkan ${ }^{\mathrm{a}}$, Orhan Öztürk ${ }^{\mathrm{b}, *}$, Ahmet E. Eroğlu ${ }^{\mathrm{c}}$ \\ ${ }^{a}$ Materials Science and Engineering Program, Izmir Institute of Technology, Urla 35430, Izmir, Turkey \\ ${ }^{\mathrm{b}}$ Department of Physics, Izmir Institute of Technology, Urla 35430, Izmir, Turkey \\ ${ }^{\mathrm{c}}$ Department of Chemistry, Izmir Institute of Technology, Urla 35430, Izmir, Turkey
}

Received 1 March 2005; accepted in revised form 13 May 2005

Available online 22 June 2005

\begin{abstract}
In this study, a medical grade CoCrMo alloy was coated with TiN by means of physical vapor deposition (PVD) technique at $550{ }^{\circ} \mathrm{C}$ for 6 $\mathrm{h}$. The TiN layer microstructure and thickness were studied by X-ray diffraction (XRD) and cross-sectional scanning electron microscopy (SEM). The adhesive strength of the TiN coatings on the CoCrMo substrate was studied by a commercially available Scanning Scratch Tester. Static immersion test was conducted to investigate the effectiveness of TiN coating in preventing the dissolution of metal ions into the simulated body fluid (SBF) from the substrate by atomic absorption spectrometry (AAS) and inductively coupled plasma optical emission spectrometry (ICP-OES). The XRD results showed that the PVD coated TiN films exhibited (111) preferred orientation, while the SEM analysis indicated quite uniform and highly dense TiN coated layer (about $3 \mu \mathrm{m}$ thick) with a columnar growth mode reaching from substrate to coating surface. The scratch test results showed that the adhesive strength between the TiN film and the CoCrMo substrate was adequate. The AAS and ICP-OES results showed that the presence of the TiN coating prevented the release of cobalt and chromium metal ions from the substrate CoCrMo alloy whereas cobalt was preferentially dissolved from the as-polished material. Calcium phosphate precipitation was observed on the surface of the as-polished material, indicating a degree of bioactivity of the as-polished surface which is absent in the TiN coated substrate alloy.
\end{abstract}

(C) 2005 Elsevier B.V. All rights reserved.

Keywords: Cobalt-chromium alloy; TiN; Immersion test; Atomic absorption spectrometry; X-ray diffraction; Scanning electron microscopy; Scratch testing

\section{Introduction}

CoCrMo alloys are highly biocompatible materials [1,2] and are widely used as orthopedic implant materials in clinical practice such as hip joint and knee replacement due to its superior mechanical properties, good wear and corrosion resistances. The biocompatibility of CoCrMo alloy is closely related to its excellent corrosion resistance due to the presence of an extremely thin passive oxide film that spontaneously forms on the alloy surface. XPS analysis reveals that its composition is predominantly $\mathrm{Cr}_{2} \mathrm{O}_{3}$ oxide with some minor contribution from $\mathrm{Co}$ and Mo oxides [3,4]. These films also form on the surfaces of other metallic biomaterials (stainless steels, titanium and its alloys) and

\footnotetext{
* Corresponding author. Tel.: +90 232750 7513; fax: +90 2327507509 .

E-mail address: orhanozturk@iyte.edu.tr (O. Öztürk).
}

serve as a barrier to corrosion processes in alloy systems that would otherwise experience very high corrosion rates [5].

In spite of the excellent corrosion resistance of CoCrMo alloy, there is still a concern about metal ion release from orthopedic implants into the body fluids (serum, urine, etc.). Implant components fabricated from $\mathrm{Co}-\mathrm{Cr}$ based alloys have been reported to produce elevated $\mathrm{Co}, \mathrm{Cr}$ and $\mathrm{Ni}$ concentrations in body fluids [6,7]. Metals from orthopedic implant materials are released into surrounding tissue by various processes, including corrosion, wear and mechanically accelerated processes such as stress corrosion, corrosion fatigue and fretting corrosion. Such metal ions and wear debris, concentrated at the implant-tissue interface, may migrate through the tissue. Research shows that the metal release is associated with clinical implant failure, osteolysis, cutaneous allergic reactions and remote site accumulations [6]. 
It is found that the surface oxide film of the $\mathrm{Co}-\mathrm{Cr}$ inhibits the dissolution of metal ions but is not always stable in the human body. Hanawa et al. [8] characterized the surface oxide films formed on CoCrMo alloy during immersion in various biological environments, Hank's solution, cell culture medium and incubation with cultured cells. Cobalt was dissolved during immersion in the Hank's solution, cell culture medium and incubation in a cell culture and after its dissolution the surface oxide consisted of chromium oxide containing a small amount of molybdenum oxide.

One approach for preventing and/or reducing the potentially harmful metal ion release from orthopedic implant materials is to coat the surfaces of these materials. Physical vapor deposition (PVD) is a well-known surface treatment method and provides a commercially viable means of depositing thin coatings of $\mathrm{TiN}$ and $\mathrm{TiC}$, especially without detriment to the substrate mechanical properties. These nitrides that have an excellent combination of performance properties are used in cutting tools, steels and medical devices. Titanium nitride (TiN) is used mostly in medical devices and metallic biomaterials due to its greater inertness to the corrosive body fluids than the metallic substrate.

Wisbey et al. [9] investigated the metal ion release from TiN coated (via PVD) CoCrMo surgical implant material. In this study, metal ion release was detected in saline solution $\left(0.17 \mathrm{M} \mathrm{NaCl}\right.$ and $2.7 \times 10^{-3} \mathrm{M}$ EDTA $)$ by means of atomic absorption spectroscopy (AAS). The results of this study indicated that significant concentrations of $\mathrm{Co}, \mathrm{Cr}$ and $\mathrm{Mo}$ were released into the solution from the uncoated specimens, while the presence of the TiN coating substantially reduced the concentration of dissolved metal ions, particularly cobalt.

Besides TiN, other types of coatings are considered to be beneficial for preventing the metal ion release. Hsu and Yen [10] investigated the metal ion release and corrosion resistance of $\mathrm{ZrO}_{2}$ thin coating on the dental $\mathrm{Co}-\mathrm{Cr}$ alloys. In this research, dental $\mathrm{Co}-\mathrm{Cr}$ alloy was electrolytically deposited with $\mathrm{ZrO}_{2}$ ceramic coating. A dynamic polarization test was used to compare the corrosion resistance of the $\mathrm{ZrO}_{2}$ coated and uncoated $\mathrm{Co}-\mathrm{Cr}$ alloys in artificial saliva. After the dynamical polarization test the metal ion release, especially $\mathrm{Co}$ and $\mathrm{Cr}$, was analyzed by graphite furnace atomic absorption spectroscopy (GFAAS). The results of GFAAS showed that the amount of Co released was greater than that of $\mathrm{Cr}$ and the metal ion release from the $\mathrm{ZrO}_{2}$ coated specimens was much less than that from the uncoated specimen.

Goldberg and Gilbert [11] investigated the electrochemical and mechanical behavior of passivated and TiN/AIN coated CoCrMo and Ti6Al4V alloys. In this study, a set of CoCrMo and Ti6Al4V alloy samples were passivated with nitric acid, while another set of specimens were coated with a novel TiN/AlN coating (via PVD). The electrochemical measurements were made in phosphate buffer solution
(PBS) at $\mathrm{pH}=7.0$. This study found that the TiN/AlN coating had a higher hardness and modulus of elasticity than CoCrMo and Ti6Al4V. It was much less susceptible to fracture, had higher interfacial adhesion strength, and was a better barrier to ionic diffusion than the surface oxides on CoCrMo and Ti6Al4V. Liu et al. [5] investigated the electrochemical performance of PVD TiN coated Ti-6Al$4 \mathrm{~V}$ artificial hip joints. In this study, TiN coatings deposited on $\mathrm{Ti}-6 \mathrm{Al}-4 \mathrm{~V}$ substrates using a plasma assisted electron beam PVD technique were electrochemically tested in $0.5 \mathrm{~N}$ $\mathrm{NaCl}$ solution. This study found that the PVD TiN coatings significantly reduced the corrosion rate. Bolton and $\mathrm{Hu}$ [12] attempted to reduce corrosion damage that may occur with metal on metal implants by applying hard ceramic coatings to surgical grade $\mathrm{Co}-\mathrm{Cr}-\mathrm{Mo}$ alloy. They found that $\mathrm{TiN}$ coatings (produced by evaporative PVD) were highly effective in preventing corrosion provided that they were thick enough to produce complete coverage.

In the present study, we investigate the effectiveness of PVD coated TiN layers on medical grade CoCrMo alloy in preventing the dissolution of metal ions into the simulated body fluid (SBF). Our study combines the use of X-ray diffraction (XRD), cross-sectional scanning electron microscopy (SEM), atomic absorption spectroscopy (AAS) and inductively coupled optical emission spectroscopy (ICP-OES).

\section{Experimental}

Medical grade CoCrMo alloy (ISO 5832-12), provided by a local company (HIPOKRAT A.S., Izmir, Turkey), was the base material with nominal compositions of $26 \% \mathrm{Cr}, 6 \%$ Mo and balance Co (all in wt.\%). The specimens had a disclike geometry with a diameter of $3.0 \mathrm{~cm}$ and a thickness of $0.3 \mathrm{~cm}$. Before TiN deposition, all the specimens were polished to a mean surface roughness of about $0.05 \mu \mathrm{m}$ based on surface profilometry. The samples were ultrasonically cleaned in alkaline Extran solution and high purity water for $15 \mathrm{~min}$ at $40{ }^{\circ} \mathrm{C}$, dried, and stored under clean room conditions. TiN coatings were deposited by using a commercial CemeCon PVD Unbalanced Magnetron Sputtering coating system. The system consists of four vertically opposed rectangular magnetrons in a closed field configuration. Before deposition, the substrate CoCrMo alloy was sputter etched with argon to remove impurities. The deposition was carried out in an argon and nitrogen atmosphere. During deposition, the pressure was kept constant at $0.5 \mathrm{mPa}$, discharge voltage was $110 \mathrm{~V}$, the substrate temperature was $550{ }^{\circ} \mathrm{C}$, and the deposition time was $6 \mathrm{~h}$. A total of six as-polished CoCrMo substrates were coated and they were used for the various characterization techniques.

X-ray diffraction (XRD) was done in the symmetric $\theta / 2 \theta$ Bragg-Brentano mode using a Philips X'pert XRD system in a step-scanning mode $\left(0.05^{\circ}\right.$ step size and $4 \mathrm{~s}$ count-time 
per step) to investigate coating-induced phases. Scanning electron microscopy (SEM) was used to determine the coating thickness and to investigate the coating surface morphology both before and after in vitro tests described below.

The adhesive strength of TiN films to the CoCrMo substrates was studied by using Shimadzu scanning scratch tester, SST-W101. The working principle of this tester is similar to that of a record-player cartridge, swinging on and scanning the specimen surface. The moving-magnet type cartridge vibrates horizontally (in the $x$-direction) above the surface of a sample, while a progressively increasing normal load is applied to the sample. Simultaneously with the start of the loading and the increase in the normal load, the sample is moved with constant speed linearly (in the $y$ direction). A transducer mounted to the diamond indenter device is used to record the response of the coating surface. The transducer output voltage can be used to interpret the processes between the diamond tip and coated surface. The experiments performed with this tester are based on the estimation of the film adhesion to the substrate by determining a critical load at which the film starts to spall or to delaminate. More details of the scratch tester and critical load measuring principles can be found in Ref. [13]. The scratch testing parameters used in this study were the following: full test force $196 \mathrm{mN}$, loading speed $5 \mu \mathrm{m} / \mathrm{s}$, and scratch speed $20 \mu \mathrm{m} / \mathrm{s}$. Three scratch tests were performed for one of the TiN coated samples. After the scratch tests, the scratch traces within the coating were inspected by SEM.

Static immersion tests were performed with the aspolished and TiN coated specimens in simulated body fluid (SBF): $\mathrm{NaCI}(6.547 \mathrm{~g} / \mathrm{L}), \mathrm{NaHCO}_{3}(2.268 \mathrm{~g} / \mathrm{L}), \mathrm{KCI}(0.372$ $\mathrm{g} / \mathrm{L}), \mathrm{Na}_{2} \mathrm{HPO}_{4}(0.124 \mathrm{~g} / \mathrm{L}), \mathrm{MgCl}_{2} \cdot 6 \mathrm{H}_{2} \mathrm{O}(0.305 \mathrm{~g} / \mathrm{L})$, $\mathrm{CaCl}_{2} \cdot 2 \mathrm{H}_{2} \mathrm{O}(0.368 \mathrm{~g} / \mathrm{L}), \mathrm{Na}_{2} \mathrm{SO}_{4}(0.071 \mathrm{~g} / \mathrm{L})$. The SBF was buffered at physiological pH 7.4 at $37^{\circ} \mathrm{C}$ with $50 \mathrm{mM}$ Tris(hydoxymethyl)aminomethane and $36.23 \mathrm{mM} \mathrm{HCI}$ acid. Sodium azide $\left(\mathrm{NaN}_{3}, 1 \mathrm{~g}\right)$ was also added into the SBF for preventing microbial effects. The ion concentrations of the SBF were similar to the human blood plasma and are summarized in Table 1. Before the static immersion test, the uncoated sides of the test specimens were coated with epoxy resin so that the metal ion release will be detected from the coated areas only. The areas that were exposed to the SBF

Table 1

Ion concentration (mM) of simulated body fluid and human blood plasma

\begin{tabular}{lrc}
\hline Ion & SBF & Human blood plasma \\
\hline $\mathrm{Na}^{+}$ & 142.0 & 142.0 \\
$\mathrm{~K}^{+}$ & 5.0 & 5.0 \\
$\mathrm{Mg}^{2+}$ & 1.5 & 1.5 \\
$\mathrm{Ca}^{2+}$ & 2.5 & 2.5 \\
$\mathrm{Cl}^{-}$ & 147.8 & 103.0 \\
$\mathrm{HCO}_{3}^{-}$ & 4.2 & 27.0 \\
$\mathrm{HPO}_{4}^{2-}$ & 1.0 & 1.0 \\
$\mathrm{SO}_{4}^{2-}$ & 0.5 & 0.5 \\
\hline
\end{tabular}

were 16.50 and $9.90 \mathrm{~cm}^{2}$ for the as-polished and TiN coated specimens, respectively. To check the efficiency of the epoxy, an uncoated (as-polished) sample was coated completely with it and exposed to the static immersion test as the other specimens. A total of six test specimens were involved in the immersion test (two TiN coated, two aspolished, and two epoxy coated). All the test specimens were incubated in SBF at $37^{\circ} \mathrm{C}$. After the immersion, $5 \mathrm{~mL}$ samples were removed from incubation bottles (each initially containing $130 \mathrm{~mL}$ immersion test solution and a test specimen) to be analyzed for the metal ion release periodically. The time interval for solution removal $(5 \mathrm{~mL}$ each time) was chosen to be $1,3,7,15$ and 30 days and then each month thereafter. The static immersion test duration was 5 months in total. At the end of this period, the immersion test specimens were removed from the solution, gently rinsed with distilled water and dried at room temperature. The morphological and elemental analyses of the test specimens were performed by SEM in secondary electron mode (SE) and energy dispersive X-ray mode (EDX).

$\mathrm{Co}, \mathrm{Cr}$ and $\mathrm{Ni}$ ion release into the $\mathrm{SBF}$ was monitored via electrothermal atomic absorption spectrometry (ETAAS) using Thermo Elemental Solaar M6 series atomic absorption spectrometer, while Ti and Mo ion release into the SBF was monitored by inductively coupled plasma optical emission spectrometry (ICP-OES) using Varian Liberty Series II ICPOES. Single element standard solutions of $\mathrm{Co}, \mathrm{Cr}, \mathrm{Ni}$, $\mathrm{Mo}$ and $\mathrm{Ti}$ were prepared from their respective stock standards (Merck Inc., $1000 \mathrm{mg} / \mathrm{L}$ ). Although no difference was observed between the calibration sensitivities (slopes of the calibration plots) of the aqueous and matrix-matched standards, the latter plot was used in all quantitative determinations. (In the preparation of matrix-matched standards, the required amount of elemental stock solution was taken and diluted to the mark with SBF. At least five standard solutions were used in the generation of calibration plots.) The analytical detection limits for $\mathrm{Co}, \mathrm{Cr}$ and $\mathrm{Ni}$ were found to be $0.5 \mu \mathrm{g} / \mathrm{L}$, while they were 30 and $20 \mu \mathrm{g} / \mathrm{L}$ for $\mathrm{Ti}$ and Mo, respectively.

During the immersion test, the $\mathrm{pH}$ values of the solutions were also monitored and they were found to vary between 7.4 and 7.6.

\section{XRD results and discussion}

Fig. 1 shows the XRD results for one of the TiN coated specimens. Included in the same figure are the results for the as-polished sample (the substrate material). In Fig. 1, the substrate (CoCrMo) peaks are labeled as " $\gamma(h k l)$ " for the fcc $\gamma$-(CoCrMo) phase and " $\varepsilon(h k l)$ " for the hop $\varepsilon$ (CoCrMo). Based on the intensity of the XRD peaks the volume percent of the substrate $\gamma$ phase is estimated to be about $90 \%$, while the rest $(10 \%)$ is due to the substrate $\varepsilon$ phase. The lattice parameter for the substrate $\gamma-(\mathrm{CoCrMo})$ 


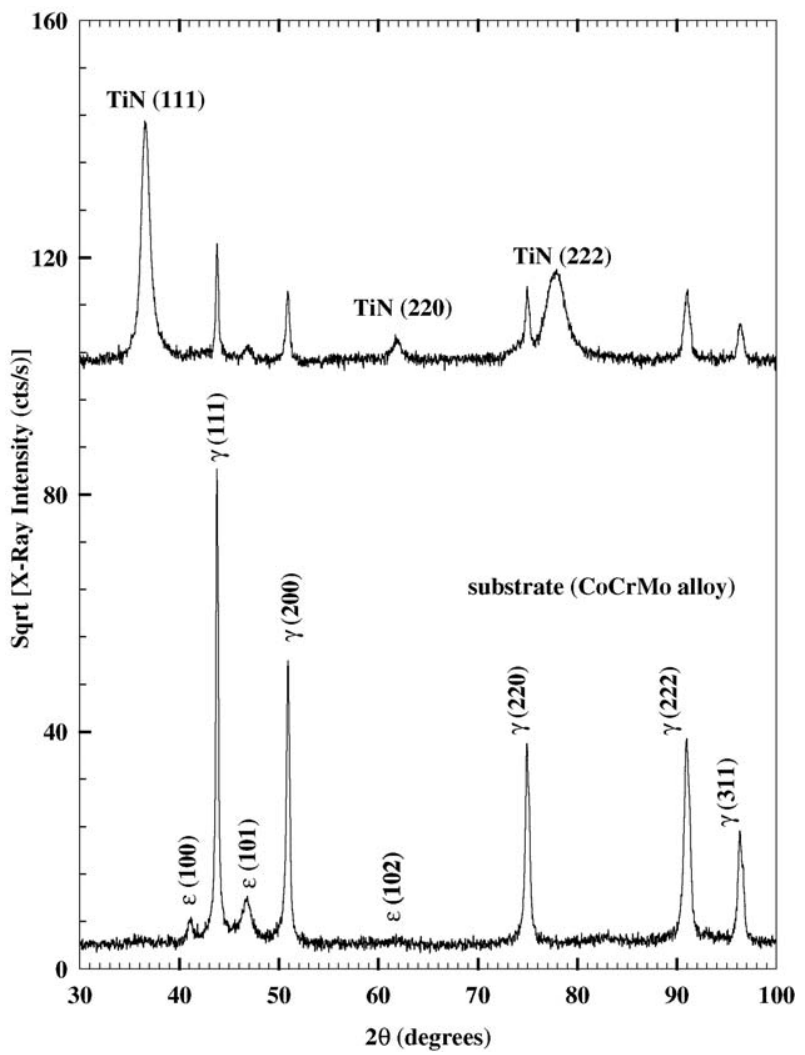

Fig. 1. XRD data for the as-polished and TiN coated specimens. Note that the square root of the intensity is plotted to reveal more clearly the weaker peaks.

phase was calculated and found to be $3.58 \AA$. The lattice parameters, a and $\mathrm{c}$, for the substrate $\varepsilon$-(CoCrMo) phase was $1.32 \AA$ and $2.70 \AA$, respectively. The XRD analysis of the TiN coated specimens revealed the presence of only three distinct peaks associated with TiN phase that is fcc structured. From the XRD data, it can be seen that the TiN film has the preferred orientation of $\mathrm{TiN}\langle 111\rangle$, which shows a similarity to those TiN films prepared by vacuum arc deposition and nitrogen ion dynamic mixing implantation methods [14-16]. The lattice constant for the TiN layer was calculated from the XRD data and was found to be $4.240 \AA$. This value is quite close to the value found in Ref. [15].

The formation of (111) preferred orientation has its origin of kinetically controlled growth. The (111) plane is the most closely packed and exhibits the lowest surface energy. If no ionic additional bombardment occurs during the process, TiN crystallites develop with random orientation, into columnar microstructure.

\section{SEM results and discussion}

SEM analysis was done on one of the TiN coated specimens and the results are shown in Fig. 2. Fig. 2(a) represents the cross-sectional view of the TiN film and quite clearly reveals the uniform nature of the TiN coated layer with a well-defined interface between the coated layer and the CoCrMo substrate. The interface indicates good coating to substrate adhesion. The cross-sectional view of the TiN film exhibits columnar structure reaching from substrate to the coating surface. The SEM picture given in Fig. 2(b) shows the surface morphology of the TiN film and, as it can be seen from this image, the film is highly dense and quite uniform. However, there appears some sort of surface defects, micro-metal droplets, on the film surface. These droplets sputtered into the structure of the TiN film may be associated with the deposition process, during which only two of the four cathodes in the deposition chamber were working. Micro-metal droplets have been shown to encourage corrosive attack in TiN coating by providing a path through which the corrosive environment could penetrate to the substrate material [12].

\section{Scratch test results and discussion}

The adhesive strength of thin films to substrates is characterized by the critical load at which the coating fails. Fig. 3 shows the load-cartridge output curves recorded
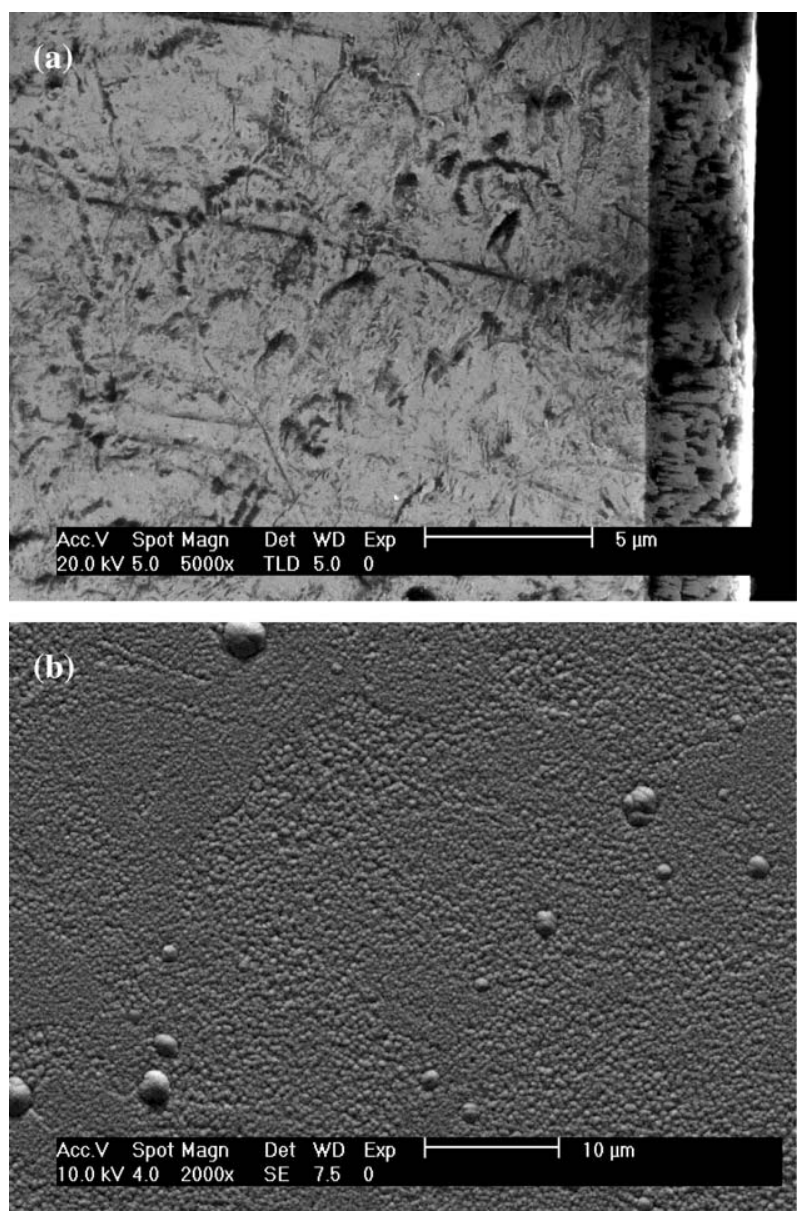

Fig. 2. Cross-sectional SEM image for (a) the TiN coated specimen; the SEM image in (b) refers to the surface morphology of the TiN coated specimen. 

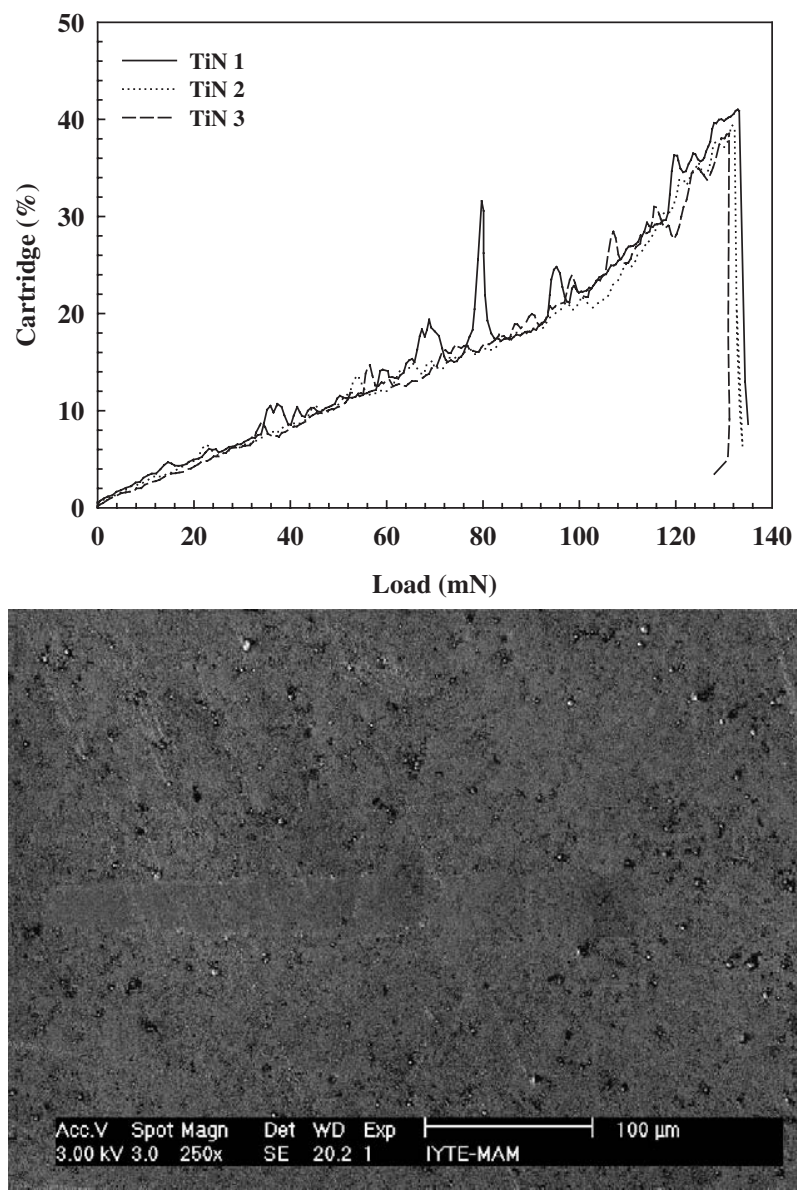

Fig. 3. Scratch resistance profiles for one of the TiN coated CoCrMo alloys. The SEM image shows a typical scratch produced on the surface of the TiN coated CoCrMo sample.

during scanning scratch tests on the same TiN coated specimen and demonstrates the scratch resistance behavior of the TiN film on the CoCrMo substrate alloy (a total of three scratch profiles obtained from the same TiN coated specimen). In theses curves, a sudden increase in cartridge output (referring to the transducer output voltage [13]) is related to damage of the coating and thus to the critical load, where the coating fails. Also included in the same figure are the SEM results of a typical scratch produced on the surface of the TiN coated CoCrMo sample. Based on the scratch profiles in Fig. 3, the average critical load was found to be $107 \mathrm{mN}$, which suggests sufficient adhesive strength of TiN film to the CoCrMo substrate. A study by Peng et al. [17], which investigated the structural and mechanical properties of TiN coatings deposited on cemented carbide tools (CrWMn steel) by pulsed high energy plasma technique, found that the adhesive strength of TiN film to the substrate was satisfactory with the highest critical load up to more than $90 \mathrm{mN}$ (in this research, the adhesive strength of the films was measured by a nanoindenter with a nanoscratch attachment). Goldberg and Gilbert [11] also investigated the adhesive strength of both passivated and TiN/AIN coated CoCrMo and Ti6Al4V alloys. In Ref. [11], the minimum load required to produce a detectable scratch was defined as a measure of the strength of the coating. Their results show that a higher load $(\sim 0.4 \mathrm{~N})$ was required to plastically deform the surface of the passivated CoCrMo compared to that of the passivated Ti6Al4V. For the TiN/AlN films, the lack of any measurable scratches produced in the surfaces of the coated specimens with loads of up to $0.6 \mathrm{~N}$ indicates that these loads were not high enough to produce plastic deformations in the coating surface.

We believe that the critical load value found in this study indicates strong adhesion of TiN films to the CoCrMo substrate and this agrees quite well with the qualitative observation based on the cross-sectional SEM results (Fig. 2). Further evidence supporting the good adhesion quality of the films in this study comes from an investigation by Tek et al. [18] which studied the mechanical properties of TiN coatings on $\mathrm{Cr}-\mathrm{Ni}$ alloy. The TiN coatings in Ref. [18] were deposited using the same coating system that was also used in this study and the deposition conditions were similar. They found that the hardness of the TiN coated samples increased by about 8 times compared to the uncoated samples and the ball-on-disc wear testing showed that the wear resistance of TiN process applied surfaces was significantly improved. Research [17] suggests that stronger adhesion of TiN films to the tool substrate will cause samples to have a better wear resistance.

\section{Static immersion test results and discussion}

The total concentrations (in $\mu \mathrm{g} / \mathrm{cm}^{2}$ ) of the metal ions released into the body fluid after 150 days were monitored by AAS and ICP-OES. The AAS and ICP-OES results indicate that the $\mathrm{Co}, \mathrm{Cr}, \mathrm{Mo}, \mathrm{Ni}$ and $\mathrm{Ti}$ metal ion release levels from the test specimens coated with TiN are found to be well below the analytical detection limits (the detection limits of $\mathrm{Co}, \mathrm{Cr}$ and Ni by GFAAS were $0.5 \mu \mathrm{g} / \mathrm{L}$ for all the three elements while those of Mo and Ti by ICP-OES were 30 and $20 \mu \mathrm{g} / \mathrm{L}$, respectively). On the other hand, detectable concentration of cobalt $\left(0.63 \mu \mathrm{g} / \mathrm{cm}^{2}\right)$ was released into the solution from the uncoated as-polished test specimen; the presence of the coating substantially reduced the concentration of dissolved metal ions. These findings suggest that the TiN coating can be an effective barrier for reducing the potentially harmful ions, in particular Co from the substrate material (CoCrMo alloy).

The metal ion release may be associated with both the film structure and film thickness. In Ref [9], which is a similar metal ion release study to ours, it is reported that the TiN coating substantially reduced the concentration of dissolved metal ions, particularly cobalt; however, the coating was less efficient for reducing molybdenum ion release than that of cobalt and chromium. This was explained by the porous structure of the film and incomplete surface coverage. Pores on a film can act as a channel for corrosive media and increase the metal ion release from the 
substrate. In our study, the film structure is quite dense and no pores were observed on the surface of the film. Another reason for the metal ion release differences between this study and the one in Ref. [9] may be related to the TiN film thickness, which was $2 \mu \mathrm{m}$ for the PVD coated films in Ref. [9], while the TiN coated layer thickness was $\sim 3 \mu \mathrm{m}$ in this study. The thicker film is probably much more effective in reducing the metal ion release.

The total concentration of the cobalt ions released into the simulated body fluid from the as-polished material $(0.63$ $\mu \mathrm{g} / \mathrm{cm}^{2}$ ) was estimated from the AAS data shown in Fig. 4. This figure shows the cobalt ion release behavior as a function of the immersion time for the as-polished substrate material. As can be seen from this figure, the as-polished sample exhibits a rapid release of Co in SBF during the initial period of the immersion test duration (first 7 days). After longer time exposures, a small increase in cobalt ion release was observed in SBF. The relatively rapid ion release for the first 7 days suggests that there are significant ion release processes occurring as soon as the as-polished specimens are immersed into the SBF.

Metal ion release into the SBF from the as-polished specimens coated with epoxy resin was also found to be well below the analytical detection limits. The results for the epoxy coated polished specimens suggest that the epoxy coating can also be an effective physical barrier for reducing the metal ion dissolution from the substrate material. The results also indicate that the epoxy coating prevented the metal ion dissolution from the uncoated sides of the TiN coated disk specimens.

After the immersion test, the surface morphology of the TiN coated and as-polished test specimens were investigated by scanning electron microscopy (SEM) as well as visually. The visual inspection of the as-polished test specimens after the immersion test shows that about $50 \%$ of the surface appears to be covered with a white type of formation. There were no such formations on the surfaces of the TiN coated test specimens examined visually after the immersion test. The SEM results after the immersion test are shown in Fig. 5. The SEM image in Fig. 5(a) shows almost full coverage

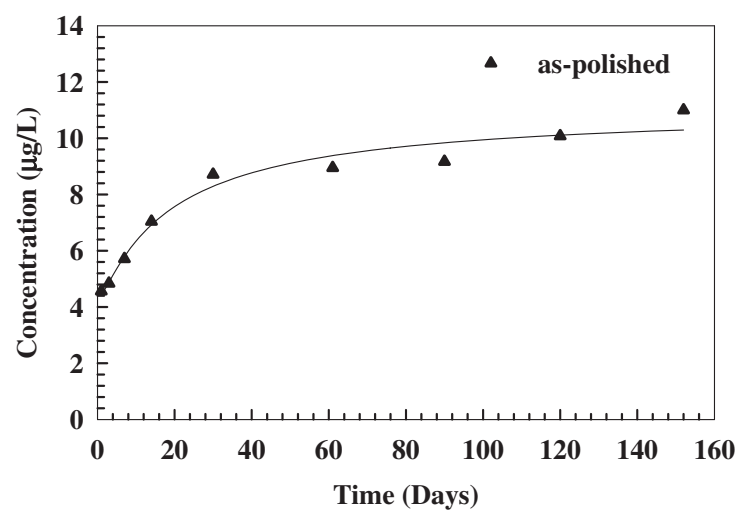

Fig. 4. AAS data corresponding to cobalt concentration released from the as-polished CoCrMo alloy as a function of time.
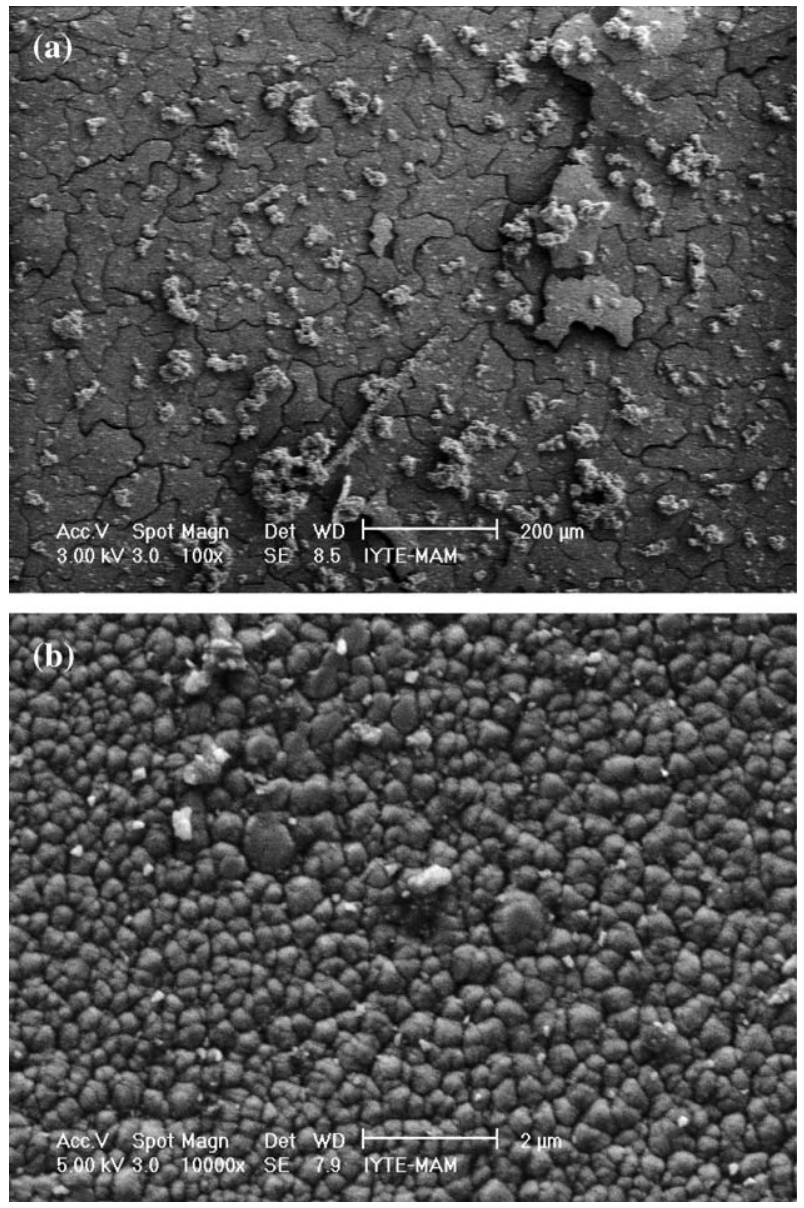

Fig. 5. SEM images related to surface morphology of (a) the as-polished and (b) TiN coated specimens after static immersion test.

of the as-polished surface with what it looks like mineral deposits, which appear slightly cracked but firmly adherent to the substrate. These results suggest that the precipitation took place during the immersion test. The EDX analysis of these deposits (precipitates) reveals calcium and phosphate peaks and indicate that the precipitates are strongly enriched in calcium (Ca) and phosphorus (P). The quantitative EDX analysis results find a ratio of calcium to phosphate of 1.65 , which indicates that the deposits are hydroxyapatite (HA)like (HA is the main mineralic constituent of bones and teeth). This finding agrees quite well with a previous investigation (via XPS) that also showed the calcium phosphate precipitation on CoCrMo alloy in Hank's solution (with compositions similar to those in SBF) and the culture medium and incubation in a cell culture $[8,19]$.

The SEM results related to the surface morphology of the TiN coated test specimen after the immersion test is shown in Fig. 5(b). As can be seen from this SEM image, there was almost no precipitation taking place on the surfaces of the TiN coated specimens during the immersion test. There were only sparsely distributed few regions (tiny white spots) where the precipitations (containing $\mathrm{Ca}$ and $\mathrm{P}$ ) might have formed. However, by means of the EDX analysis the contents of these precipitations were found to be not so 
enriched in calcium and phosphorus compared to those found on the as-polished material. Our finding does not seem to agree with a literature study [19], which, by means of scanning electron microscopy, reveals the presence of a phase of calcium phosphate on TiN coated hip prosthesis heads explanted postmortem after a minimum of 5 years from arthroplasty. In order to understand the reasons for the observed bioactivity, Piscane et al. in Ref. [20] looked into the details of the oxidation process of TiN crystals, and studied the initial nucleation of calcium phosphate on the oxidized TiN surface by a combination of spectroscopic (XPS) and first principles molecular dynamics techniques. Their results indicate that TiN surfaces are able to induce the spontaneous nucleation of calcium phosphates if they are only partially oxidized.

In order to understand the lack of bioactivity observed in the TiN films in this study, a detailed analysis of oxide layer(s) formed on the TiN coated layers both before and after the static immersion tests are necessary. There is no conclusive evidence on the oxide film formation on the coated layers based on the experimental findings here. Also, a detailed investigation of structure and composition of the films is necessary since the chemical behavior of TiN films strongly depends on them [20].

HA-like deposits observed on the as-polished CoCrMo substrate alloy in this study suggest signs of bioactivity of the CoCrMo alloy, which is absent in the TiN coated samples. Bioactivity is widely accepted as the essential requirement for an artificial biomaterial to exhibit chemical bonding to living tissues upon the formation of a bone-like apatite layer on its surface in any simulated body environment. A bioactive ceramic coating, especially HA coating, on metallic orthopedic alloys could be a promising approach to shortening the time for osseointegration and improving bone-bonding ability, as well as reducing the release of potentially harmful metal ions from metallic implants. There exists a large body of literature that focuses on the development of bioactive coatings by dissolution of calcium and phosphate from simulated body fluids [21-24]. Garcia et al. [22] investigated the development and characterization of coatings containing bioactive glass particles by the solgel method on 316L stainless steel. In this study hybrid coatings of silica containing hydroxyapatite, bioactive glass and glass-ceramic particles were prepared and applied on metal substrate. The coated samples were tested in vitro to study their electrochemical properties and bioactive response. Their results show that the coatings improve the corrosion resistance of the steel substrate and in vitro tests (in simulated body fluid) revealed that all the films show signs of bioactivity. In another sol-gel coating study by Kim et al. [23] hydroxyapatite (HA) was coated onto a Ti substrate with the insertion of a titania $\left(\mathrm{TiO}_{2}\right)$ buffer layer. In this study, the HA layer was employed to enhance the bioactivity of the Ti substrate, while the $\mathrm{TiO}_{2}$ intermediate layer was inserted to improve the bonding strength between the HA layer and Ti substrate (since the bonding strength of
HA coatings on metallic materials are relatively low, usually an intermediate layer between metal and HA could be helpful for improving the interfacial bonding condition [24]), as well as to prevent the corrosion of the Ti substrate. The results of this study showed that the insertion of the $\mathrm{TiO}_{2}$ layer significantly improved the bonding strength of the HA layer to the Ti substrate. The results also showed that the corrosion resistance of $\mathrm{Ti}$ was enhanced by the presence of $\mathrm{TiO}_{2}$ coating, as confirmed by potentiodynamic polarization tests.

\section{Conclusions}

The effectiveness of TiN coating in preventing the dissolution of metal ions into the simulated body fluid (SBF) from the substrate was investigated. The XRD and SEM results for the PVD coated specimens indicated that the TiN coatings exhibited (111) preferred orientation and had a coating thickness of $3 \mu \mathrm{m}$ with a columnar type of growth mode. The scratch test results indicate adequate adhesive strength between the TiN film and the CoCrMo substrate. The AAS and ICP-OES results showed that the release of potentially harmful metal ions, in particular cobalt and chromium from the substrate CoCrMo alloy, was prevented by the presence of the TiN coating. The AAS analysis showed that the cobalt was preferentially dissolved from the as-polished material. The results suggest that the TiN coating is an effective barrier to metal ion release compared to the surface oxides on the CoCrMo substrate alloy. The SEM analysis results after the immersion test showed there was calcium phosphate precipitation (HA-like deposits) on the surface of the as-polished material, indicating a degree of bioactivity of the as-polished surface which was absent in the TiN coated substrate material.

\section{Acknowledgments}

The authors are grateful to Hipokrat A.S. for supplying medical grade CoCrMo alloys and to Prof. Ahmet Öztarhan of Ege University, Izmir, Turkey, for carrying out the TiN coatings. Prof. Sebnem Harsa is acknowledged for the contributory discussions. We also thank Dr. Mustafa Toparli of Metallurgical and Materials Engineering Department at Dokuz Eylül University for performing the scratch tests. Thanks also go to Mr. Sinan Yilmaz and Mrs. Oya Altungöz for their help with the AAS and ICP-OES analyses. This work is partially funded by Izmir Institute of Technology through research grant IYTE 2003/14.

\section{References}

[1] K.S. Katti, Colloids Surf., B Biointerfaces 39 (2004) 133.

[2] M. Long, H.J. Rack, Biomaterials 19 (1998) 1621. 
[3] I. Milošev, H.-H. Strehblow, Electrochim. Acta 48 (2003) 2767.

[4] A. Kocijan, I. Milošev, B. Pihlar, J. Mater. Sci., Mater. Med. 15 (2004) 643.

[5] C. Liu, Q. Bi, A. Matthews, Surf. Coat. Technol. 163-164 (2003) 597.

[6] Y. Okazaki, E. Gotoh, Biomaterials 26 (2005) 11.

[7] J.J. Jacobs, A.K. Skipor, L.M. Patterson, N.J. Hallab, W.G. Paprosky, J. Black, J.O. Galante, J. Bone Joint Surg. 80-A (1998) 1447.

[8] T. Hanawa, S. Hiromoto, K. Asami, Appl. Surf. Sci. 183 (2001) 68.

[9] A. Wisbey, P.J. Gregson, M. Tuke, Biomaterials 8 (1987) 477.

[10] H.C. Hsu, S.K. Yen, Dent. Mater. 14 (1998) 339.

[11] J.R. Goldberg, J.L. Gilbert, Biomaterials 25 (2004) 851.

[12] J. Bolton, X. Hu, J. Mater. Sci., Mater. Med. 13 (2002) 567.

[13] E. Schaffer, G. Kleer, Surf. Coat. Technol. 133-134 (2000) 215.

[14] T. Matsue, T. Hanabusa, Y. Ikeuchi, Vacuum 74 (2004) 647.
[15] L.A. Rocha, E. Ariza, J. Ferreira, F. Vaz, E.R. Beriro, L. Rebouta, E. Alves, A.R. Ramos, Ph. Goudeau, J.P. Riviere, Surf. Coat. Technol. $180-181$ (2004) 158.

[16] B. Škorić, D. Kakaš, N. Bibic, M. Rakita, Surf. Sci. 566-568 (2004) 40.

[17] Z. Peng, H. Miao, L. Qi, S. Yang, C. Liu, Acta Mater. 51 (2003) 3085.

[18] Z. Tek, M.A. Gungor, M. Sonugelen, C. Artunc, A. Oztarhan, Surf. Coat. Technol. 196 (2005) 317.

[19] T. Hanawa, Mater. Sci. Eng., C 24 (2004) 745.

[20] S. Piscanec, L.C. Ciacchi, E. Vesselli, G. Comelli, O. Sbaizero, S. Meriani, A. De Vita, Acta Mater. 52 (2004) 1237.

[21] P. Ducheyne, Q. Qiu, Biomaterials 20 (1999) 2287.

[22] C. Garcia, S. Cere, A. Duran, J. Non-Cryst. Solids 348 (2004) 218.

[23] H.-W. Kim, Y.-H. Koh, L.-H. Li, S. Lee, H.-E. Kim, Biomaterials 25 (2004) 2533.

[24] T. Wang, A.D. Reisel, J. Mater. Sci. 39 (2004) 4309. 